\title{
COMMISSION 8: POSITIONAL ASTRONOMY (ASTRONOMIE DE POSITION)
}

PRESIDENT: L. V. Morrison

VICE-PRESIDENT: T. E. Corbin

ORGANIZING COMMITTEE: P.Benevides-Soares, L. Helmer, Hu Ning-sheng,

J Kovalevsky, L. Lindegren, J. A. Lopez, M. Miyamoto, F. Noël,

D. Polojentsev, Y. Requieme, H. Schwan, M. Yoshizawa

\section{Introduction}

The scope of this report embraces activities in wide-angle optical astrometry, and specifically excludes photographic astrometry, which is the province of Commission 24, and radio-astrometry, which is the province of Commission 40.

This somewhat arbitrary sub-division of positional astronomy by discipline rather than subject is not particularly satisfactory scientifically, and this point is being addressed by the General Secretary who has expressed the wish to merge commissions.

The report is based on information supplied by members of the Commission. Where no information was forthcoming, I tried to compensate for the deficiency by adding material from other sources. Nevertheless, there are areas where the report fails to do justice. For this I apologise.

References to papers are quoted in square brackets where they are listed in Astronomy $\&$ Astrophysics Abstracts; otherwise simplified abbreviations for journals are used, as recommended by Commission 5 .

\section{Meetings, Symposia, Colloquia}

Problemy astrometrii: Kosmicheskoj geodimamikj, Kiev, Ukraine, September 1990 [54.012.049].

IAU Colloquium 127, Reference Systems, Virginia Beach, USA, October 1990 [53.012.086].

Linkage of terrestrial, dynamical, stellar and extragalactic reference frames (III), Japanese Symposium on Earth Rotation, Astrometry and Geodesy, Naruko, Japan, January 1991 [53.012.079].

IAU Symposium 156, Developments in astrometry and their impact on Astrophysics and Geodynamics, Shanghai, China PR, Sept. 1992, (eds. Mueller \& Kolaczek), Kluwer Acad. Press.

Galactic and Solar System Optical Astrometry: Observation \& Application, Cambridge, UK, June 1993, (eds. Morrison \& Gilmore), Cambridge Univ. Press. 


\section{Ground-based astrometry}

ARGENTINA. The Repsold meridian circle of the Felix Aguilar Astronomical Observatory continued its programme of observation of fundamental stars, and the Fourth San Fernando Meridian Circle Catalogue was published [54.041.026]. Observations of 16 minor planets were compiled in order to study the behaviour of the FK5 around $-30^{\circ}$ [54.002.127].

BRAZIL. Astrolabe observations continued at Rio Grande and Valinhos. The Second Rio Grande Catalogue of 122 FK4 and FK4S stars by the Danjon OPL01 astrolabe was published [53.002.091]. Optical positions of the radio star $\alpha$ Scorpii by the astrolabe at Valinhos were published [53.041.036].

The reduction of meridian circle observations by an overlap method was tested [53.041.034], and the colour-index and mag. dependence of declinations from three astrolabe catalogues observed at Valinhos were investigated [53.041.037].

CHILE. The Repsold Meridian Circle at Santiago continued its programme of fundamental stars and published the Second Santiago Declination Catalogue of 493 FK5 stars [54.002.031]. The Santiago Fundamental Catalogue of 1105 FK5 stars (J2000.0) was published (A\&AS,95,355). The astrolabe at Santiago continued its programme of radio stars, and positions of $\alpha$ Scorpii and 9 Sagitarrii were published (A\&AS,96,567).

CHINA(PR). Photoelectric astrolabes and transit instruments continued in use at the Observatories of Beijing, Shanghai, Shaanxi, Yunnan and Purple Mountain. Their programmes centered on extending the fundamental optical reference frame to fainter magnitudes and linking the opti$\mathrm{cal}$ and radio frames. A new type III astrolabe (A\&A,271,360) was installed at Xin Long station, Beijing Observatory. The low latitude meridian circle of Yunnan Observatory was being manufactured, and the horizontal meridian circle built co-operatively by Shaanxi and Copenhagen Observatory was adjusted optically: and mechanically.

In December 1991 the type II photoelectric astrolabe of Beijing Observatory was transferred to San Juan, Argentina, to participate in the southern astrolabe programme. In November 1992 the type II photoelectric astrolabe of Shanghai Observatory was moved to Beijing Observatory.

The following photoelectric astrolabe catalogues were published: Third Beijing PACP3 [52.002.081], Second General Catalogue of 2606 stars GCPA2 [55.002.131], Third General Catalogue of 2577 stars GCPA3 [53.002.106], Second Yunnan [53.041.033], Fourth Preliminary Beijing PACP4 [55.002.132], Third PPCP3 and Fourth PPCP4 Beijing [54.002.060, 54.002.061], Yunnan 606 geodetic stars [54.041.008].

The Purple Mountain Catalogue of right ascensions of 508 stars obtained with the photoelectric transit instrument [55.041.014], and an analysis of the General Catalogue (CGS) of 4949 geodetic stars [55.041.017] were published. Precise positions and proper motions of 1980 General Catalogue stars were obtained [54.002.123].

\section{DENMARK, GREAT BRITAIN \& SPAIN}

The Carlsberg Automatic Meridian Circle (CAMC) continued in regular use at the international observatory of the Roque de los Muchachos on the island of La Palma in the Canaries. It was operated jointly by Copenhagen University Observatory, the Royal Greenwich Observatory and the Real Observatorio y Instituto de la Armada en San Fernando.

The new and more accurate micrometer designed by Helmer which was fitted to the CAMC in March 1988 was descibed in [54.034.107]. This new micrometer was first used in the production of Carlsberg Meridian Catalogue Number 5 [53.002.129]. It contains positions and magnitudes of $\sim 17000$ stars north of $-45^{\circ}, \sim 15000$ proper motions and 2847 positions of 66 solar system 
objects. The mean error of a catalogue position in the zenith is 0.07 in right ascension and declination and 0.04 in magnitude. The same accuracy applies to Carlsberg Meridian Catalogue Number 6 [55.002.071] which contains positions and magnitudes of $\sim 12800$ stars, $\sim 11600$ proper motions, and 1368 positions and magnitudes of 69 solar system objects.

Carlsberg Meridian Catalogues 1-6 contain 28 000 of the $\sim 36000$ International Reference Stars north of $-45^{\circ}$. Catalogues $5 \& 6$ contain the beginning of the observation $(\sim 6400)$ of the $\sim 36000$ faint reference stars $(\mathrm{V} \sim 12)$ selected at Copenhagen University Observatory and the Real Instituto y Observatorio de la Armada from the Astrographic Catalogue zones between $-45^{\circ}$ and $+90^{\circ}[53.041 .053]$.

In October 1991 the divided circle was replaced by a new glass circle from Heidenhain with Diadur division lines. The six photoelectric cameras for reading the declination circle were replaced by CCD cameras. Using these new cameras in fast read-out mode enabled the telescope to be set more accurately, and stars were routinely scanned in the smallest slits which are 12" long.

A joint discussion of the CAMC observations of FK5 stars with those made at Bordeaux was published [52.041.011]. This provided evidence of systematic errors in the FK5 reaching 0.1 in some parts of the sky. Another joint discussion with Bordeaux - the optical positions of 23 radio stars - was published [52.041.004]. The first meridian circle observations of Pluto were published (A\&A,262,347).

Positions of 53 radio stars obtained with the San Fernando Meridian Circle (SFMC) were published [53.041.054]. Following this programme the SFMC was taken out of service and automated, with a similar scanning slit and operational system as the Carlsberg AMC. Discussions began to find a suitable site in South America for this telescope.

FRANCE. Since 1990 the Bordeaux automatic meridian circle was mainly used for the determination of faint reference star positions from the Faint Reference Star list selected by the Carlsberg group in the range $11<\mathrm{V}<12$. Measurements of 8000 stars in the zones +10 to +25 and +50 to $+76 \mathrm{deg}$. have been completed. Positions of 221 radio stars were published [54.041.004]. Some of these were compared with VLA radio positions and the alignment of the FK5 and radio frames was deduced [54.041.024].

The global treatment of the Bordeaux AMC observations was investigated [52.041.028]. Taking as reference the preliminary Hipparcos positions of FK5 stars obtained by NDAC and FAST Consortia after processing of 18 months of data, a new reduction of eight years of Bordeaux differential observations was carried out. The results showed a remarkable improvement of the reference star residuals, with, however, small systematic waves (amplitude 0"04) corresponding to unexpected systematic errors.

Development of a CCD micrometer was started and it was planned to mount it on the Bordeaux AMC in early 1994. Measurements of faint stars down to 15th magnitude could then be measured with an accuracy of $0.02-0.03$, observing in narrow strips and taking as reference the final Hipparcos positions of stars with $V>9$ when they were released.

Improvements of the prototype photoelectric astrolabe were discussed and a new programme of observation with respect to FK5 was started [52.041.021]. The catalogue of stars observed with the CERGA photoelectric astrolabe March 1988 to July 1991 was published (A\&AS,96,477). Systematic deformations of the apparent almucanter as derived from Danjon astrolabes in Paris and Santiago-de-Chile were investigated (A\&AS,274,621). The result of eight campaigns of astrolabe observations of the radio star $\beta$ Persei were published (A\&AS,87,513). 
GERMANY. The FK5 extension, comprising 3117 stars down to magnitude 9.5 taken from the FK4S and IRS, was compiled by the Astronomisches Rechen-Institut and US Naval Observatory [55.002.039].

Work continued on the data base ARIGFH at the Astronomisches Rechen-Institut. The main aim is to collect all the relevant astrometric data on stellar positions and proper motions in a comprehensive data bank. During the period of this report the main task was to bring older catalogues into a machine-readable form. There are now more than 1400 of such astrometric catalogues available, 1200 of which have been typed into the computer at Heidelberg. The crossidentifications of all these observations with the stars in the master catalogue and the formation of the data base was started.

ITALY. The programme of observation with the astrolabe of the Cagliari Astronomical Observatory continued, and the positions of radio stars were published [53.041.047].

JAPAN. The Tokyo Photoelectric Meridian Circle (Tokyo PMC) continued in operation. The third and fourth annual catalogues (PMC87, PMC88) of 5748 and 3800 stars, respectively, in the FK5 system were published [53.002.142, 55.002.152]. The global adjustment of star positions for the compilation of independent catalogues was investigated [53.041.031]. Systematic errors in declination arising from secular changes in the graduated circle were also investigated [53.041.045].

Work continued on developing a CCD micrometer for the PMC. Test observations were carried out on the Mitaka Gautier meridian circle. The first experimental model, used in drift-scanning mode (DISC=DIgital Strip scanning Ccd), enabled magnitude 14.5 to be reached. Work started on a second version (DISC-II), using a larger chip $(\sim 1200 \times 1200$ pixels $)$ with reduced read-out noise which would permit 16 magnitude to be reached. It was planned to stop the first PMC programme in 1993 and replace the photoelectric micrometer by DISC-II. The second PMC programme of observation would bègin by strip-scanning the declination band -25 to +35 (IAU Symp.156, 71).

A method of improving fundamental star catalogues using astrolabe observations was applied to the Mizusawa observations of FK4 stars [52.041.004].

LATVIA. The catalogues, Ri-80 and Ri-86, containing the right ascensions of stars observed for the time service of the University Observatory, were published [53.002.041].

POLAND. Systematic differences in zenith distance from two successive groups of stars in the OPL astrolabe at Borowice were investigated [54.041.016]. The influence of standard and anomalous refraction on astrolabe results was also investigated [54.041.019].

ROMANIA. An analysis was carried out of the separation in right ascension of wide doubles observed with the Bucharest Meridian Circle [54.041.017].

RUSSIA. At Pulkovo Observatory the Zverev Photographic Vertical Circle (PVC) after its reconstruction in 1987, carried out $\sim 8000$ absolute observations of 2050 FK5 star declinations from the North Pole to $-15^{\circ}$. It was planned to complete the first version of the PVC catalogue in 1994.

The absolute declination PVC catalogue which was compiled from 12762 observations made in Chile in 1964-66 was finished and made ready for publication. It contains $\sim 700$ FK4 stars and 700 FKSZ stars (IAU Symp.141, 68).

In 1990 the observations of two star lists with the Pulkovo Horizontal Meridian Circle (HMC) were finished. The Catalogue of 170 reference stars around 63 radiosoures - Lazorenko list - 
and the Catalogue of 911 stars (502 FK5 and 409 FKSZ stars) were compiled and published. Also the results of the differential observation of declinations with the HMC made in 1982-83 were finished and published.

At the Kislovodsk Mountain Station $(\mathrm{h}=2100 \mathrm{~m})$ regular meridian observations of the Sun, Mercury, Venus, Mars and daytime stars were made using the Struve-Erthel Vertical Circle and Struve-Erthel Large Transit Instrument. In 1990-1992 more than 1500 observation of planets and Sun and about $\mathbf{4 7 0 0}$ observations of stars were made with an accuracy more than twice as good as that obtained with the same instument in Pulkovo.

Work continued on new meridian instruments and modernising old ones. Experiments with CCD micrometers were carried out with the Struve-Erthel Great Transit Instrument on Kislovodsk Mountain Station (IAU Coll.136), with the Pulkovo Suharev HMS (IAU Symp.156, 119). Experimental CCD-observations with short-focus telescopes were started in 1990 as a part of the development of the REGATTA-ASTRO space astrometry project (Space Research Institute and Pulkovo Observatory). Astrometric and photometric results obtained with very small CCD-cameras $(D=80, F=212 \mathrm{~mm}$ ) shows that they are suitable for the purpose.

More precise simultaneous astrometric and photometric CCD-observations with a short-focus astrograph $(D=100, F=712)$ were started in December 1992 at Pulkovo. Preliminary results of differential observations (exposure $2.5 \mathrm{~min}$., field $0.8 \times 1.1 \mathrm{deg}$.) were as follows: accuracy of position determination, $0 " 1$ to $0 " 3$ for stars from 8 to 14 mag. (series of 5 frames with 2.5 min. exposure); accuracy of photometry, 0.02 to 0.1 for a single observation (frame).

A new Pulkovo Meridian Reflecting System by A.A.Nemiro and Yu.S.Strletsky was in the process of being developed.

The construction of the Meridian Automatic Horizontal Instrument (MAHIS) was well advanced. In 1991-92 a new mirror for the MAHIS was made from carbide silicon. All optical and mechanical parts of the MAHIS were built in triplicate during 1990-1993. Some investigations of MAHIS were carried out (IAU Symp.156, 117).

Two papers concerning some problems of the determination of systematic errors in declination, especially of the FK5 catalogue were prepared for publication in the Izvestia GAO. Also considered was the problem of the influence of reference catalogue errors on the parameters of the fundamental system using minor planet observations. The analysis and generalization of the methods of compilation of secondary reference systems using meridian observations were carried out. The methods for unification of meridian catalogues, which were created without the strict principles of differential connection to the fundamental system, were investigated.

A detailed comparative analysis of two general catalogues of bright stars (BSC and KGZ) was completed. The work of amalgamating these two catalogues was preparatory to obtaining the united catalogue of positions and proper motions of 6474 stars down to 7.9 mag. in the FK5 system.

The absolute catalogue of declinations of 249 stars based on the Sanders-Raimond method and observed with the Sternberg Institute GOMZ's transit circle and zenith telescope was published [52.002.100]. The external accuracy was estimated to be $\pm 0 " 19$ [52.002.101]. 
UKRAINE. As part of the CONFOR programme, the positions of 2149 stars around 238 radio sources were observed with the Nikolaev Repsold meridian circle. Observations of Uranus and Neptune were continued. The Sun and planets were observed with two instruments: FreibergKondratiev transit instrument in Nikolaev: Sun (145 observations), inner planets (136), external planets (29); Struwe-Erthel large transit instrument at Kislovodsk Mountain Station (see above).

Nikolaev Observatory in collaboration with Pulkovo finished observation of two differential catalogues made with the Pulkovo Horizontal Meridian Circle (IAU Symp.156, 119). The $\mathrm{Pu}$ (HMC)88 catalogue consists of 911 selected stars from FK5B, FK4S and FKFS, of which 502 belong to the FK5. It was shown that the systematic accuracy of the Pulkovo HMC was $\sim 0 " 02-0 " 03$. It was confirmed that the FK5 has systematic errors of about 0.1 in the declination zone +40 to +60 . The $\mathrm{Pu}(\mathrm{HMC}) 89$ catalogue, consisting of 170 AGK3 reference stars around 63 extragalactic radio sources, was published in VINITI, 1992.

The semi-automization of the Nikolaev Repsold meridian circle was finished. Four CCD matrices were used for the circle-reading, having an accuracy of $\pm 0 " 05$ for one microscope. Two computers were used for the registration and reduction of the observations. The Axial Meridian Circle (AMC) underwent investigation and automization. The previous value of the flexure was 0.09 . Collimation stability with temperature was 0.026 per $1^{\circ} \mathrm{C}$. The systematic errors of the AMC were not expected to be more than 0"'02. (Pinigin et al. , 1993, in Kinematics and Physics of Celestial Bodies, in press).

The Nikolaev Observatory took part in the joint project MAHIS, involving Pulkovo and Kazan Observatories. Nikolaev Observatory finished the meteorological data collection and time service for MAHIS (IAU Symp.156, 117).

At Kiev the orientation of the FK5 and VLBI frames was investigated using the positions of 251 radio sources [54.043.002, 54.043.003]. The RRS2 list of 2575 meridian reference stars in the fields of 238 radio sources was prepared [55.002.065]. The observation of the position of planets continued at the Kiev State University Astronomical Observatory [55.041.015].

USA. The U.S. Naval Observatory continued with its program to produce an absolute catalog of observations of the FK5, IRS and radio stars from pole to pole. The observations made with the 6-inch transit circle in Washington, DC and the 7-inch transit circle on Black Birch, New Zealand were over $90 \%$ complete. Large numbers of observations of solar system objects were being made as part of that program. The 7-inch catalog of 23000 stars observed in 1967-73 was compared with the FK4 [55.041.008].

The positions of 12 FK5 stars obtained with the Mark III optical interferometer were published [52.041.006]. The accuracies were 10 mas in RA and 6 mas in Dec. The Navy Prototype Optical Interferometer was in the process of being constructed at Anderson Mesa, Flagstaff, Arizona. First fringe detection was expected in late 1993, with observing commencing in the spring of 1994. The first program would be aimed at producing a catalog of $1000+$ bright stars with accuracies of better than 4 mas.

The observations made with the 6-inch transit circle in Washington from 1977 to 1982 were being reduced to form the W1J00 catalog. The instrumental system was compared with the FK5, showing differences less than $150 \mathrm{~ms}$ of time in RA and 180 mas in Dec. Equator and equinox points were determined with respect to DE200.

The compilation of the Washington Fundamental Catalog (WFC) was in progress. Most of the effort was concentrated on the determination of zero points of the WFC system using observations of the Sun, Mercury and Venus. These data were taken from the absolute catalogs observed since 1900. Investigations into the possible use of observations of the minor planets, 
Mars and Jupiter to strengthen the solution were also being carried out. Error estimates in astrometric catalogues were investigated [52.002.091].

The work on the W1J00 and WFC zero points was also used to evaluate W. Fricke's solution for the correction to the FK4 equinox. Using observations of the Sun, Mercury and Venus from eight Washington catalogs and six Cape catalogs (1907-1971), it was found that the FK5 proper motion system needed a correction of $-0 " 73 / \mathrm{cy}$. The observations of the Sun made for the W1J00 confirmed this result.

At the USNO Flagstaff Station, the modernization of the 8-inch transit circle continued, and was renamed the Flagstaff Astrometric Scanning Transit Telescope (FASTT). The Texas Instruments $800 \times 800$ thinned CCD was replaced by a CRAF/Cassini $1024 \times 102412 \mu$ pixel, thick frontilluminated CCD. A laser interferometer was installed to monitor motion in azimuth in realtime. The internal accuracy obtained with FASTT was $\sim 0 " 03$ for $\mathrm{V}<14$, rising to $~ 0 " 25$ at the limiting magnitude around 17.5. Besides instrumental development, observations of FK5 and radio stars, and QSOs were made (IAU Symp.141, 369; IAU Symp.156, 65).

The CCD/Transit Instrument (CTI) of the Steward Observatory produced relative positions of stars brighter $\sim 17$ mag. with standard errors less than $\sim 40$ mas over a test region of $8 \times 12$ $\operatorname{arcmin}[53.041 .001]$.

At the Space Telescope Science Institute a method of infinitely overlapping circles to obtain estimates of the systematic differences between fundamental astrometric catalogues was developed [55.041.013].

YUGOSLAVIA (former). A catalogue of 290 stars around 78 radio sources was produced with the large Meridian Circle of Belgrade Observatory [53.041.002]. Observation of solar system bodies continued [53.041.050; 55.041.006]. The positions of 223 PZT stars and 173 fundamental stars made with the MC in 1985-90 were analysed (A\&AS,97,417).

\section{Wide-angle space astrometry}

ESA - HIPPARCOS. The ESA astrometry satellite Hipparcos completed more than three years of high quality data collection since its launch in August 1989, before operations were terminated on 15 August 1993. After functioning for some months with only two gyros, observations were suspended in March 1993 following the failure of the fourth gyro. Although attempts were made to continue observations without gyros, a series of other problems were encountered (attributable to the particle radiation damage resulting from its highly elliptical orbit), which made further satellite operations impossible. The operational lifetime foreseen pre-launch was 2.5 years.

The Hipparcos mission comfortably exceeded all of the original mission goals, and significantly exceeded many of them. Data reductions completed the treatment of the first 18 months of data, including iterations, in both of the main data analysis teams. A first treatment of all three years of the mission data was expected by the end of 1993. Final positions, parallaxes and annual proper motions were expected to be below the 2 mas target for the great majority of stars. Photometric results were also outstanding, with accurate photoelectric photometry for all 120000 main mission objects, and all one million Tycho Catalogue objects, at 200 or so epochs throughout the observational period. Several thousand new double star systems were detected. Results from the two main reduction groups were in excellent agreement. Catalogue completion was foreseen for around 1996. Many of the results reported in the following paragraphs by the reduction consortia can be found in $A \& A, 258$, No.1(1992).

The FAST data-reduction consortium received and analysed all data collected by Hipparcos in the period 1989 November 27 to 1992 June 6. Several solutions using successively 6, 12 and 
18 months of data were computed, showing a steady and rapid increase in the precision of the results. Significant parallaxes were obtained even from the 12-month solution, and proper motions became computable at the level of the 18-month reduction.

The next phase was to perform an iteration on the 18-month data span. About 100000 stars were kept in that solution, the recognised and suspected double and multiple stars being temporarily left out of the solution, awaiting a determination of their elements. That iteration differed from the first treatment due to the fact that astrometric parameters obtained during the first treatment were used to recompute the attitude and the projections on reference great circles.

The results showed the following mean precisions for stars of magnitude 9:

1.4 mas in ecliptic longitude

1.2 mas in ecliptic latitude

$3.3 \mathrm{mas} / \mathrm{yr}$ in proper motion in longitude

$2.7 \mathrm{mas} / \mathrm{yr}$ in proper motion in latitude

1.6 mas in parallax.

Finally, the remaining 13 months of data were added to the iterated solution, showing a dramatic improvement in proper motions and a significant improvement in the three other parameters. Preliminary results for this global 31-month solution gave the following precisions for stars of magnitude 9:

1.3 mas in ecliptic longitude

1.1 mas in ecliptic latitude

$1.7 \mathrm{mas} / \mathrm{yr}$ in proper motion in longitude

$1.5 \mathrm{mas} / \mathrm{yr}$ in proper motion in latitude

1.5 mas in parallax.

In addition, the precison in determining the separation of the recognised (known or newly discovered) double stars was better than 12 mas. Most of the newly discovered double stars had separations between $0 " 15$ and $0 " 4$ and magnitude differences smaller than 3 .

The data reductions in the NDAC consortium were shared between the Royal Greenwich Observatory in Cambridge (processing of raw data, attitude determination and photometry), Copenhagen University Observatory (determination of star coordinates on great circles) and Lund Observatory (sphere solution and treatment of non-single stars). A succession of preliminary catalogues for over 100000 stars were produced, using the data collected during the first 6,12 , 18 and 30 months of the mission. These catalogues showed the expected progressive improvement in accuracy and clearly indicated that the mission goals of 2 mas average accuracy in the positions, annual proper motions and parallaxes would be reached, and in many cases exceeded, when all the data (approximately 37 months) were incorporated in the solutions.

Much effort was devoted to systematic comparisons with the results of the FAST consortium and with external data, which on the whole showed very satisfactory agreement. Calibrations and studies of the spacecraft dynamics performed by the NDAC group at the Royal Greenwich Observatory provided essential input for operating the satellite towards the end of its lifetime, when the on-board gyroscopes could not be used as intended.

The data reductions in the TDAC consortium were shared between the Observatory of Strasbourg, Astronomisches Rechen-Institut at Heidelberg, Astronomisches Institut at Tuebingen and Copenhagen University Observatory. The reductions made use of the satellite attitude and the Hipparcos reference catalogue received from NDAC. Working from a TYCHO Input Catalogue containing about the 3 million brightest stars on the sky, the star mapper signals were 
extracted and analysed in order to derive $B$ and $V$ magnitudes and astrometry for stars brighter than 12 mag.

The first year of observations from the TYCHO experiment were analysed to give a preliminary catalogue (TICR) of one million stars with an accuracy of 0.06 . Astrometric processing of the first four months of observations showed that the complete 3-yr TYCHO mission would result in positions, annual proper motions and parallaxes for these stars with an accuracy of $\sim 0.03$ rms at 11 mag., improving to 0"006 for stars brighter than 9 mag. (See Høg et al. in Galactic 8 Solar System Optical Astrometry, eds. Morrison \& Gilmore, Cambridge Univ. Press).

The INCA consortium completed its task of publishing the Hipparcos Input Catalogue in 1992 (ESA SP-1136) [55.002.054].

Volumes 1-5 of the main catalogue lists for the $\sim 118000$ stars in the final Hipparcos observing programme their positions, proper motions, magnitudes and colours, and where available, spectral types, radial velocities, multiplicity and variable information. The catalogue is composed of two main parts. The first is the survey of $\sim 52000$ bright stars which is complete to the magnitude limit in $V$ of 7.3 to 7.9 , depending on galactic latitude and spectral type. The second is the list of $\sim 66000$ 'faint' stars selected from the proposed observing programmes according to the priorities which were allocated by the Hipparcos Scientific Selection Committee.

Volume 6 (Annex 1) gives information and data for all known individual components of $\sim 14000$ double and multiple systems for which at least one component is identified in the main Hipparcos Input Catalogue (Vols. 1-5).

Volume 7 (Annexes 2-4) is an atlas of $\sim 10900$ identification charts for stars in the main catalogue.

\section{Proposals for future wide-angle astrometric space missions}

The ROEMER mission has been proposed to ESA by a group of European astronomers (see Høg \& Lindegren, in Galactic \& Solar System Optical Astrometry, eds. Morrison \& Gilmore, Cambridge Univ. Press). The proposed mission aims at accurate astrometric and photometric measurements of 100 million stars down to 18th magnitude. Based on the well-proven concepts of the ESA Hipparcos mission, but employing a vastly superior CCD detection system, it would give the positions, parallaxes and annual proper motions to 0.1-1.5 mas, depending on magnitude, and multi-colour photometry to 0.001-0.02 mag. The programme would include a complete survey of the 50 million stars brighter than $V=15.5$.

The AIST (Astrometric Integrating Space Telescope) project was proposed in 1989 at Pulkovo (Chubey et al. , IAU Symp.141, 77) as the 'second epoch of space astrometry' experiment. In essence, the design consists of two telescopes symmetrically placed around a central beam combiner unit. It will survey the positions, proper motions and parallaxes of 10-15 million stars brighter than 15th mag. In 1992-93 the computer simulation of the dynamic features of spacecraft and the numerical estimates of the level of errors of the output data were obtained. The arrangement and optical scheme and the focal plane ensemble were optimized (Chubey et al. , IAU Symp.156, 415). The plans for the mission are being developed in a collaboration between the Applied Mechanical Consortium in Krasnoyarsk, the Space Instrumentation Institute in Moscow and Space Flight Control Centre in Zelenograd, with contacts in the Hipparcos and Roemer scientific groups. 